\title{
Cleft Lip Repair: The Hybrid Subunit Method
}

\author{
Travis T. Tollefson, MD, MPH, FACS ${ }^{1}$
}

1 Department of Otolaryngology, University of California, Davis, Sacramento, California

Facial Plast Surg 2016;32:150-155.

\begin{abstract}
Address for correspondence Travis T. Tollefson, MD, MPH, FACS, Facial Plastic and Reconstructive Surgery, Department of OtolaryngologyHead and Neck Surgery, University of California, Davis, 2521 Stockton Blvd., Suite 7200, Sacramento, CA 95817 (e-mail: tttollefson@ucdavis.edu).
\end{abstract}

Abstract
Keywords
- cleft lip repair
- cleft rhinoplasty
- vermillion cutaneous
junction
- cutaneous roll

The unilateral cleft lip repair is one of the most rewarding and challenging of plastic surgery procedures. Surgeons have introduced a variety of straight line, geometric, and rotation-advancement designs, while in practice the majority of North American surgeons have been using hybrids of the rotation-advancement techniques. The anatomic subunit approach was introduced in 2005 by Fisher and has gained popularity, with early adopters of the design touting its simplicity and effectiveness. The objectives of this article are to summarize the basic tenets of respecting the philtral subunit, accurate measurement and planning, and tips for transitioning to this subunit approach.
For those surgeons who are accustomed to a rotation advancement technique (- Fig. $\mathbf{1}$ ), the transition to confidently executing the subunit approach can be challenging. ${ }^{1}$ In the following description, observations during my transition to using the subunit approach will be outlined. Fisher described an overwhelming $25 \mathrm{lip}$ and nasal points in his original description (-Fig. 2). ${ }^{2}$ Each of these has a clear purpose in regard to proportionality and symmetry; however, many surgeons may find the implied complexity daunting. Ultimately, the surgeon's eye can be trained to see shadows and light reflections on the normal perioral/nasal landmarks, making fewer markings needed (while the mind still processes the position of the other landmarks).

The subunit approach offers an additional tool with the potential for consistently excellent results while using a uniform marking and measurement technique. These attributes led me to transition to its use, along with the tenets respecting the borders of aesthetic units of the face and preservation of soft tissues. The objectives of this article are to correlate the hybrid subunit markings to those used in rotation-advancement, find similarities in the steps used if asymmetries are noted, and simplify the process of transitioning to a subunit approach.

\section{Technique}

The cleft lip repair is completed under general anesthesia with a midline oral RAE tube.
Avoidance of distortion of the lip is a priority to ensure proper markings, so an infraorbital nerve block using bupivacaine $0.25 \%$ with $1: 200,000$ epinephrine is used prior to tattooing the lip markings. The face of the maxilla, nasal spine, septal mucosa, buccal sulcus, oral commissures, nasal tip, and alae are prudently infiltrated. The hybrid subunit cleft lip repair will be described in terms of design and lip markings, flap mobilization and muscle dissection, rhinoplasty, and closure.

\section{Design and Lip Markings}

The cleft lip repair design begins by placing temporary skin tattoos (methylene blue) using a 30-gauge needle. The markings progress in the following order, which are a simplified version of the original Fisher technique ${ }^{1}$ (-Fig. 3):

1. Subnasale-the junction of the upper lip and central columella

2. Lateral columella junction with upper lip (noncleft side)

3. Lateral columella junction with upper lip (cleft side)

4. Midline vermillion cutaneous (VC) junction (trough of Cupid's bow)

Hint: follow maxillary frenulum

5 and 6. Cupid's bow peaks at the VC junction

Hint: an alcohol pad will dry the vermillion to enhance identification
Issue Theme Congenital Deformities of the Face, Head, and Neck; Guest Editor, Travis T. Tollefson, MD, MPH, FACS
Copyright $\odot 2016$ by Thieme Medical Publishers, Inc., 333 Seventh Avenue, New York, NY 10001, USA. Tel: +1(212) 584-4662.
DOI http://dx.doi.org/ 10.1055/s-0036-1582227. ISSN 0736-6825. 


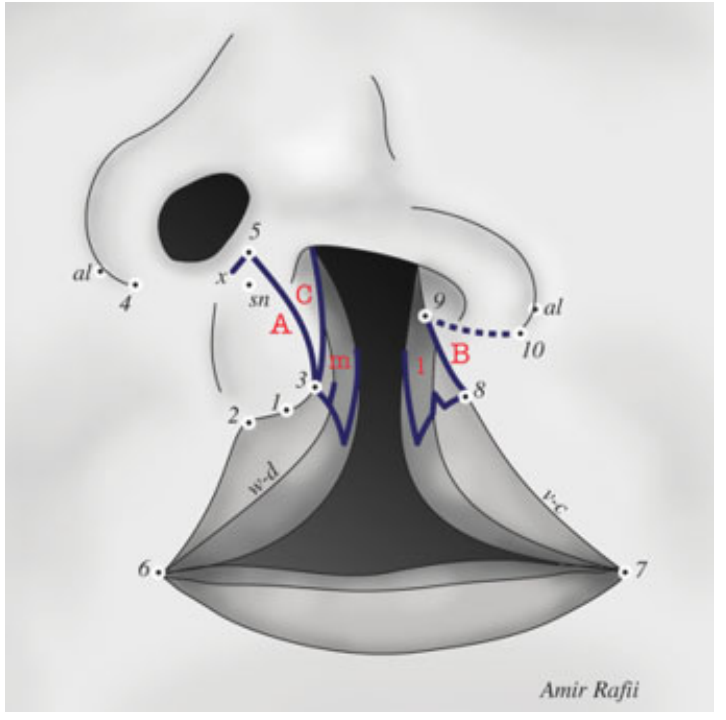

Fig. 1 Illustration of typical rotation-advancement unilateral cleft lip repair with a Mohler modification. The flaps are labeled in red as follows: $A$, rotation; $B$, advancement; $C$, columellar; $m$, medial mucosal; I, lateral mucosal. The points are labeled as follows: 1, 2, 3, Cupid's bow peak; 4 , subalar noncleft side; 5 , Mohler columellar point ( $2 \mathrm{~mm}$ up from subnasale and one noncleft one-third of columella); 6 and 7, lip commissures; 8 , Noordhoff point (inferior-most cleft-side point where the cutaneous roll and vermillion are substantial); 9 , high point on cleft side; 10 , subalar cleft side. Other markings include: al, alar; sn, subnasale; $v$-c, vermillion-cutaneous junction; $w$-d, wet-dry (vermillion-mucosal) junction.
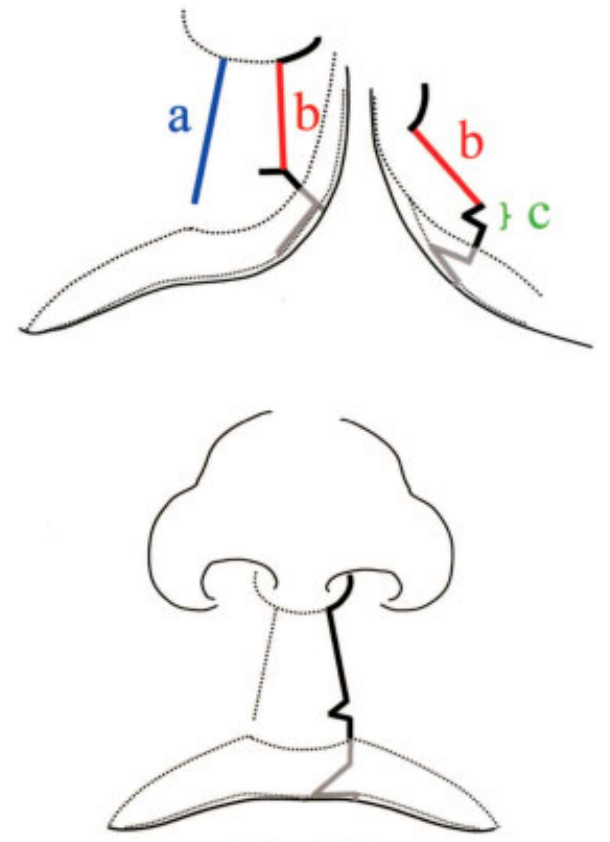

Fisher 2000

Fig. 2 The Fisher anatomic subunit cleft lip repair. (Used with permission from Fisher $^{1}$.)
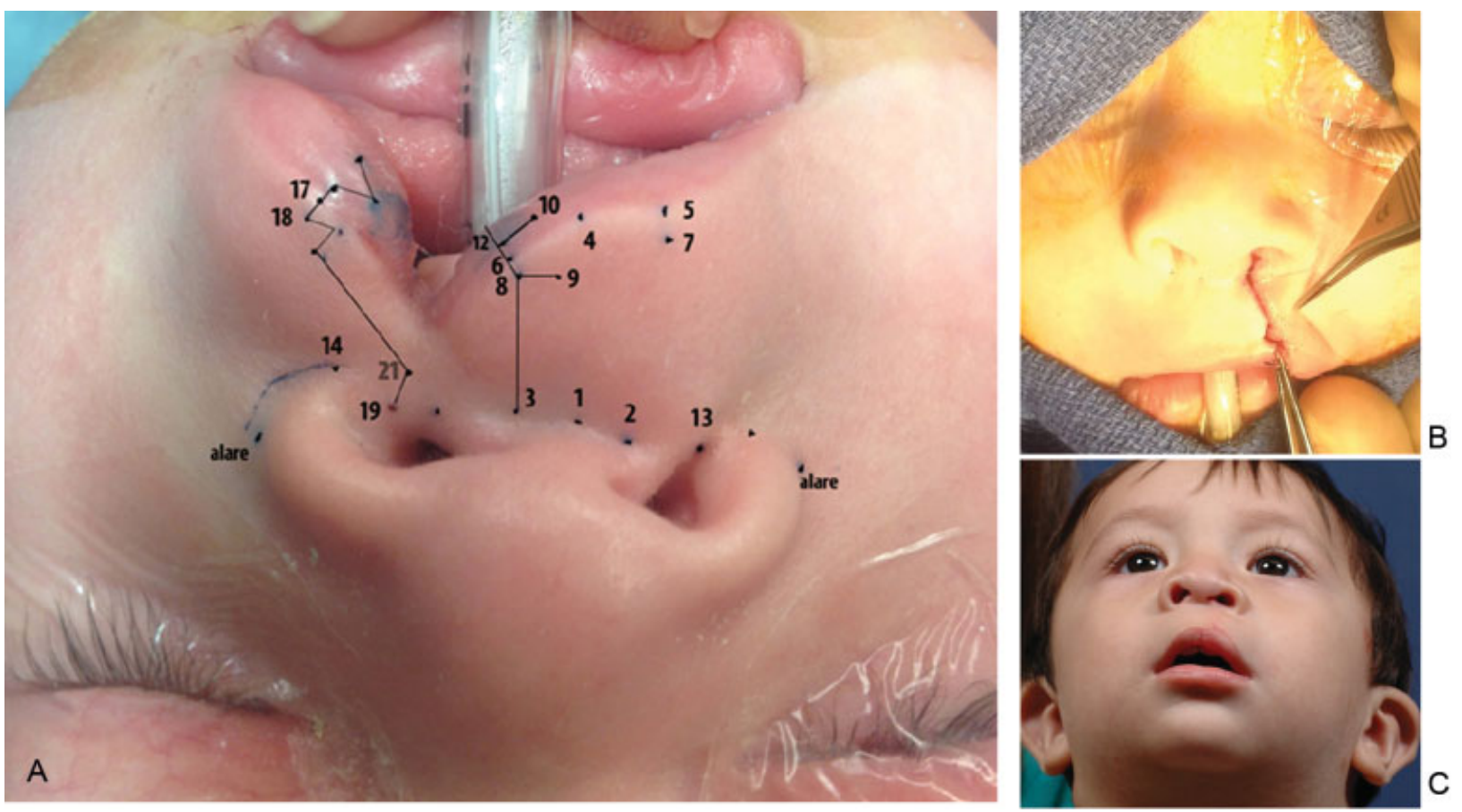

Fig. 3 Lip markings shown on an infant with an incomplete left cleft lip showing anatomic subunit approach. (A) The distance from point 2 to 7 was $11 \mathrm{~mm}$, while the distance from point 3 to 8 was $8 \mathrm{~mm}$, which makes a height difference of $3 \mathrm{~mm}$ and a lateral based triangle of $2 \mathrm{~mm}$. The distance from the subnasale to the alar on the left was $18 \mathrm{~mm}$ and $14 \mathrm{~mm}$ on the right. To prevent nostril stenosis, a conservative wedge resection of 1 to $2 \mathrm{~mm}$ at the junction of the columella-nasal sill is advised. (B) After muscle repair, the triangle flap above the cutaneous roll is placed in the recipient back cut, which is made perpendicular to the vertical height (line from points 3-8). (C) 2 years post-op from the base view demonstrating alar base symmetry. 
7 and 8. Cupid's bow peaks just superior to the cutaneous roll (site of triangle flap inset)

9. Back cut medial from 8 perpendicular to line (3-8)

10 and 12. Dry-wet lip junction midline and cleft side

Hint: cleft side-where dry lip height is similar to the noncleft side

13 and 14. Subalar-alar-lip crease at midpoint of alar insertion to lip/sill

17. Lateral lip VC junction (Noordhoff point)

18. Lateral lip just superior point 18 above the cutaneous roll

19. Uppermost lateral lip mark-closes to columellar flap (c-flap)

Hint: most difficult to choose in incomplete clefts; essential to conserve nasal sill skin to prevent nostril stenosis

21. Lateral lip segment high point where alar crease and lip meet

Hint: include normal epidermis with hairs but not nasal vibrissae

*** alar-lateral alar bases junction with the cheek

In a very similar pattern to a Mohler rotation-advancement cleft lip repair design, the hybrid subunit lip repair measurements are made in the following order:

1. Cleft-side versus noncleft-side lip height: Peak of Cupid's bow (points 7 and 8 above; the cutaneous roll) to the lateral columella/lip (points 2 and 3 )

2. Alar base discrepancy subnasale to alar bilaterally

3. Normal (noncleft side) is the normal philtral height (line from points 2-7), which is transferred with a caliper to the lateral lip starting at point 18 . The line is extended superiorly
4. The triangle at the inferior limb of the lateral lip flap edge is 1$2 \mathrm{~mm}$. (Hint: bigger triangles are more visible.) According to Fisher, the triangle design is $1 \mathrm{~mm}$ smaller than the difference between the cleft and noncleft lip heights, to account for the Rose Thompson effect. ${ }^{1}$ (Hint: If the lateral lip is short, $a$ modification of the triangle $e^{3}$ is helpful.) (-Fig. 4)

5. The lateral lip vermillion triangle flap and recipient back cut (line from points 10-12) on the cleft side are marked.

6. The back cut (from points 8-9) made just above the cutaneous roll (recipient of the lateral lip triangle flap) is made perpendicular to the philtral column line.

7. The difference in the alar base widths (alar-subnasale) is used to predict the amount of alar sill removal in an incomplete cleft lip and to set the tension of the cinching alar base suture.

Comparing the side-by-side markings of a rotationadvancement and subunit approach can be helpful in observing differences and similarities. The curvilinear rotational flap incision begins either at the subnasale (Millard) or within the columella (Mohler) in rotation-advancement techniques. ${ }^{4,5}$ In the subunit approach, the cephalad-most point of the philtral column incision is at the crease of the lateral columella/lip junction (points 2 and 3), thereby obeying the philtral subunit.

The c-flap used for either the nasal floor (Millard) or into the columella (Mohler) in rotation-advancement is altered in a subunit approach to have a half-circle shape between points 2 and 3 and laterally to the point where the columella and nasal alae meet. ${ }^{4,5}$ (Fisher point 15 and 16). ${ }^{1}$ Intraoperatively, I mark the lateral-most point of the columellar skin as it changes from skin into the cleft edge and nasal mucosa, so that this can be used for the nasal floor/sill after the nasal base

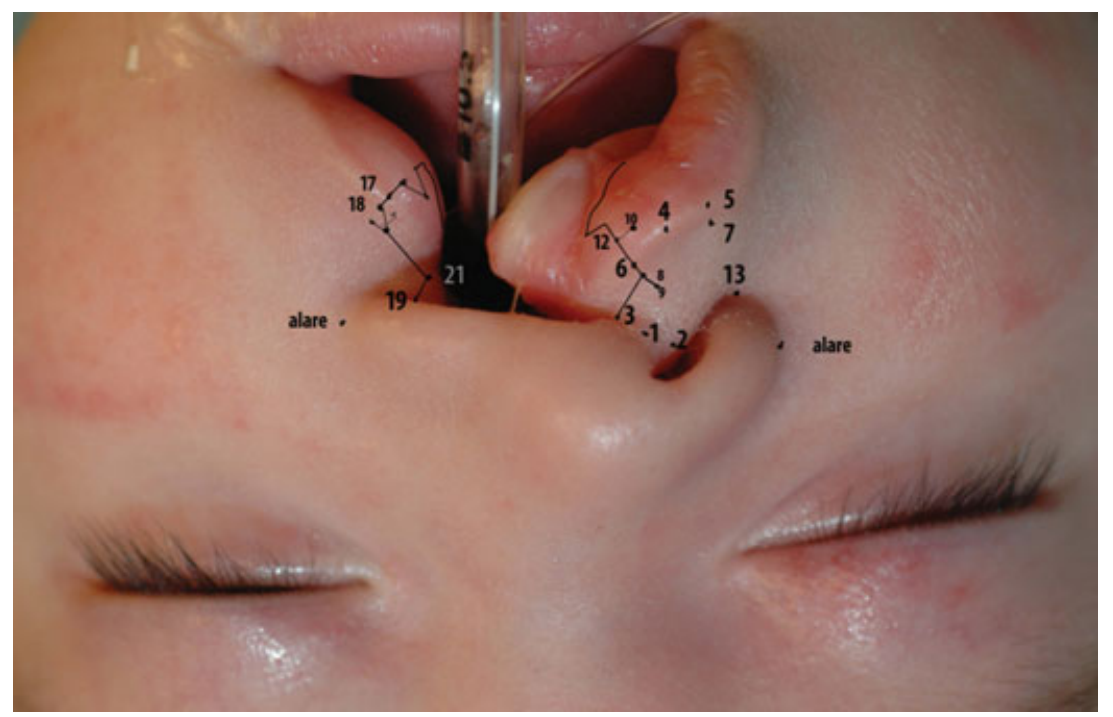

Fig. 4 Infant with a complete left cleft lip and palate showing anatomic subunit markings in place. The distance from point 2 to 7 was 11 mm, while the distance from point 3 to 8 was $7 \mathrm{~mm}$ (while stretching the lip). This difference of 4 mm suggests that a triangle that is at the extreme end of the spectrum of $3 \mathrm{~mm}$ will be needed to create the adequate lip height. Due to a short lateral lip, a Tennison modification ${ }^{3}$ is shown with the distance from point 21 to 18 of $8 \mathrm{~mm}$, while the triangle base is $3 \mathrm{~mm}$ (giving the total $11 \mathrm{~mm}$ to equal the normal noncleft-side lip height). Alar base widths of $23 \mathrm{~mm}$ on the left and $13 \mathrm{~mm}$ on the right were measured from the subnasale to alar, respectively. 


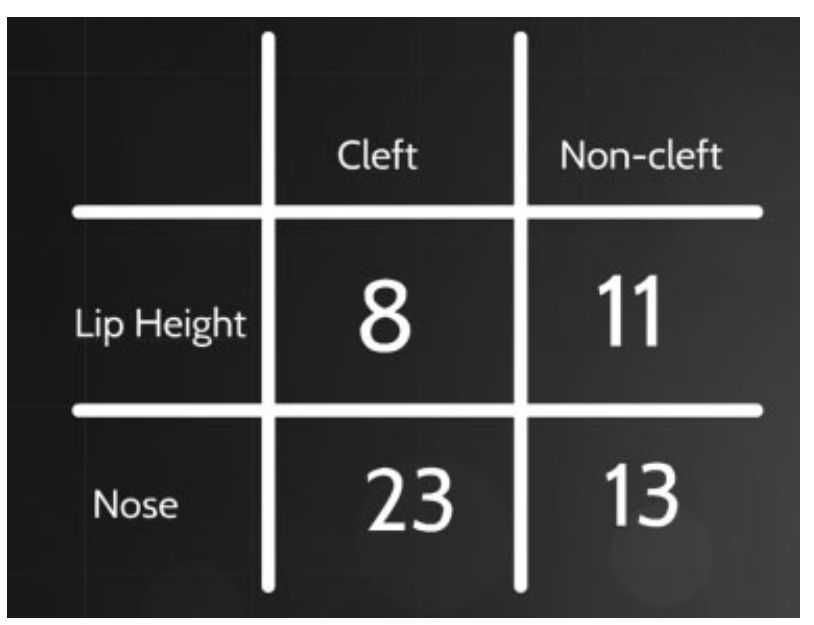

Fig. 5 Basic measurements for lip height and alar base on cleft (left) and noncleft side (right) for infant in - Fig. 4. These guide the triangle flap size and alar base width.

width is set. To adhere to the subunit principles, the lateral lip angled line will fit into the columellar nasal sill recipient, but I simply make a c-flap and then trim the size and shape. I have seen several narrow nostrils in surgeons converting to the subunit approach. (Hint: Only dermis should be designed into this "c-flap," to ensure that mucosa is not included in the skin closure.)

In a rotation-advancement approach, the cutaneous lip height is created by designing the lateral advancement flap to be equal to the distance of noncleft lip height and is measured from the columella/lip crease to Cupid's bow peak points 2 to 7. (Hint: Symmetric lip height between the noncleft and cleft side is essential.) The caliper is used to measure from points 2 to 7 on the noncleft and points 3 to 8 on the cleft side. This is recorded in a simple $2 \times 2$ table (vertical columns labeled left and right, and rows labeled lip and nose (-Fig. 5). The measured lip height of the cleft side is subtracted from the noncleft side's measurement (e.g., $9-7 \mathrm{~mm}=2 \mathrm{~mm}$ ).

With a rotation-advancement technique, this difference in lip height is addressed with a curved medial (rotation) incision that lengthens when pulled downward. Noordhoff's Chang Gung repair added a small triangle at the inferior lateral lip flap (similar to the subunit approach). ${ }^{6}$ So, if one were using a hybrid of a Noordhoff approach (as I have been for the past 10 years), then the transition from using a small triangle at the bottom of a lateral lip flap to a subunit approach is much easier. (Hint: With either approach, the small triangle flap from just above the cutaneous roll on the lateral lip segment is inserted into the inferior-most medial lip just above the cutaneous roll.)

For either the subunit or rotation-advancement approach, drawing the lateral lip design begins by picking point 17 (Hint: where the triangular-shaped vermillion and cutaneous roll are still significant). The normal philtral height (points 2-7 on the noncleft side) is transferred over to the lateral lip segment and is measured up toward the nasal sill. (Hint: Follow the alar-lip crease from lateral to medial by slightly pushing up on lip and noting the horizontal crease.) If the height needed is more than what is available without going into the nose, then the reoriented triangular design at the inferior limb is made similar to Tennison ${ }^{1,3}$ (see - Fig. 4). This triangle opens up when the lateral lip is retracted inferiorly and adds to the lip height. This is very effective at preserving the distance from the commissure to the Noordhoff point, assuring that the lateral lip subunit is not made too small.

For a rotation-advancement technique, I have always kept the design of this line as close to the cleft edge as possible to retain the normal skin (with dermal glands and small hairs present) and to preserve tissue to a possible tight closure. In the subunit approach, it has been slightly uncomfortable to "waste" some of the normal medial edge of the skin on the lateral lip. The lateral lip height design is a line that fades superomedially, but then angles toward subalar (so the lateral lip design will fit like a puzzle piece into the medial lip). For adherence to the "conservation of tissue principle," I preserve this tissue to an "l-flap" attached to the gingivobuccal sulcus to use for oral or alveolar cleft lining.

Finally, to balance the deficient dry vermillion on the cleft side to the more robust dry vermillion on the noncleft side, a small triangle flap of dry vermilion from the lateral lip segment is created to inset onto the medial lip vermilionmucosal junction (wet-dry). In some instances, the closure of the vermillion and mucosa does not allow accurate placement of this triangle and it is sacrificed.

\section{Flap Mobilization and Muscle Dissection}

After lip incision designs, the subunit and rotation-advancement techniques are relatively similar. Each surgeon has his or her preferred dissection techniques. I prefer a dissection of the lip and nasal base tissues off of the premaxilla/maxilla in a supraperiosteal plane, which is minimal for a microform cleft lip and extends to wider dissection as the severity of the cleft increases. The medial orbicularis oris fibers are released from the nasal spine/caudal septum with occasional repositioning of the septum in severe deviations. This gradated principle is used for the buccal sulcus incisions, with the nasal release being maximized by incising up to the base of the inferior turbinate in the most severe cleft nasal deformities. Regarding the extend of orbicularis oris dissection, I prefer to release 3 to $4 \mathrm{~mm}$ of the deep dermis from the orbicularis oris on the lateral segment with less dissection on the philtral side (in an effort to maintain the natural philtral dimple).

Digital grasping of the lip segments facilitates incisions and dissection. Grasping between the thumb and index finger also applies a compressive effect on the vasculature for ease of bloodless dissection. The vermillion and mucosal incisions are made with an \#11 blade. A corneal 2-mm spearshaped knife is useful for the small triangle flaps. A \#15C blade is used for the other cutaneous incisions. Regardless of the lip design technique, the mucosal flaps ( $l$ and $m$ ) are incised down to the buccal sulcus, where the medial gingivobuccal sulcus incision is continued past the frenulum medially and for approximately $2 \mathrm{~cm}$ laterally. Using a rotatory motion with a cotton-tipped applicator or gauzecovered fingertip facilitates dissection off the maxilla and 
premaxillary. (Hint: Release of the nasal base is tested by placing a medium double prong hook under the nasal base and spinning the instrument and base toward the columellar to check the residual tension.)

At the cleft margins, the orbicularis oris muscles are dissected from the underlying mucosa. (Hint: look for minor salivary glands and stay deep to them to ensure the correct plane.) Similarly, the dermis is released from the orbicularis oris muscles as described earlier.

\section{Primary Rhinoplasty}

Regardless of cleft lip design, I prefer to release the lower lateral cartilages from the skin soft tissue envelope with blunt dissection and reserve a Tajima reverse- $U$ rim incision for only the most severe nasal deformities that were not able to be prepared with nasoalveolar molding. ${ }^{7,8}$ Dissection can be safely performed through either the lateral alar base or base of columella. A round-tipped scissors (Littler are my preference) releases the medial crural foot pods and intermediate and lateral crus of the lower lateral cartilages on both cleft and noncleft side. The dissection pocket must be atraumatic to the overlying skin.

The potential for cartilage reshaping in the early neonatal period guides my interest in early repositioning. ${ }^{9}$ Typically, I use a transcutaneous plication approach, which involves passing a resorbable suture from inside the nasal vestibule through the alar-cheek crease and then back in the same puncture, allowing the knot to be inside the nose. For this to be effective, the lower lateral cartilages must be temporarily suspended cephalically (-Fig. 6). (Hint: Place a Ragnell retractor or Brown forceps with one arm in each nostril and push cephalad and ventral to make the cleft-side nasal dome equal the noncleft side.) Then, pass the through-and-through triangular fixation sutures.

Alternatively, when the above technique is not adequate, an intercartilaginous incision is created. The cephalic border

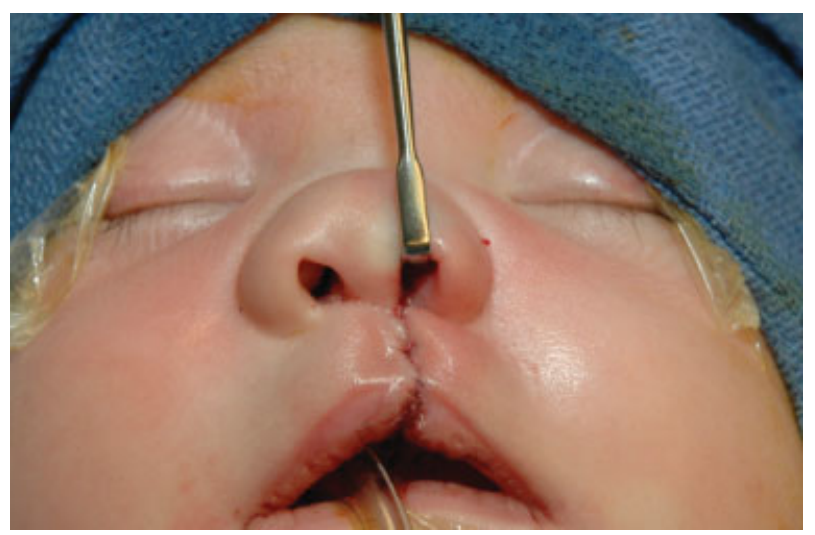

Fig. 6 Triangular fixation sutures reposition the nasal cartilages after adequate release from the skin soft tissue envelope. In this infant, after subunit repair, a Ragnell retractor is placed in the left nostril pushing the lower lateral cartilage and medially and cephalad. The Monocryl suture is passed from inside the nostril out the alar crease and back in the same hole (created by the needle). of the cleft side lateral crus of the lower lateral cartilage is suspended to the upper lateral cartilage and dorsal septum using 5-0 polydioxanone suture (PDS) sutures on a P-3 needle (similar to Skoog's description). ${ }^{10}$ In rare cases when nostril hooding is severe, a rim incision and excision as described by Tajima is used.

\section{Closure}

Closure begins with the nasal floor, ensuring that the alar base width is symmetric by using the nose measurements (subnasale to alar on right and left) from the aforementioned $2 \times 2$ table. The difference between the subunit approach and a rotation-advancement technique is that the former requires precise design planning to maintain adequate nasal sill tissue. (Hint: Early in my adoption of the subunit technique, the nasal base was narrow in several cases due to insufficient planning on my part; therefore, I began using my typical salvage of the "cflap" tissues and left the nasal sill cut until after the alar base cinching sutures were applied.)

As I improve my nasal sill and lateral lip incision designs, I have transitioned toward the subunit approach that Fisher described. If you find that some of your nasal bases are too narrow using the subunit approach, consider using this as a "training wheel" until your design is improved. Basically, I adhere to my rotation-advancement approach at the alar base where I routinely create as large of a c-flap as possible by staying at the edge of the cutaneous-mucosal margin. This flap is kept in continuity with the posterior septal mucoperichondrial flap, which is used to make the nasal floor of adequate dimensions. The floor closure prepares for the alar base "cinching" suture, which is placed between the cleft-side alar base tissues and perichondrium/soft tissues of the nasal spine and caudal-most septum. Calipers measure for symmetry from the subnasale to the alar base tattoos.

Closure of the mucosa and buccal sulcus differentially advances lateral tissue toward the midline. A midline

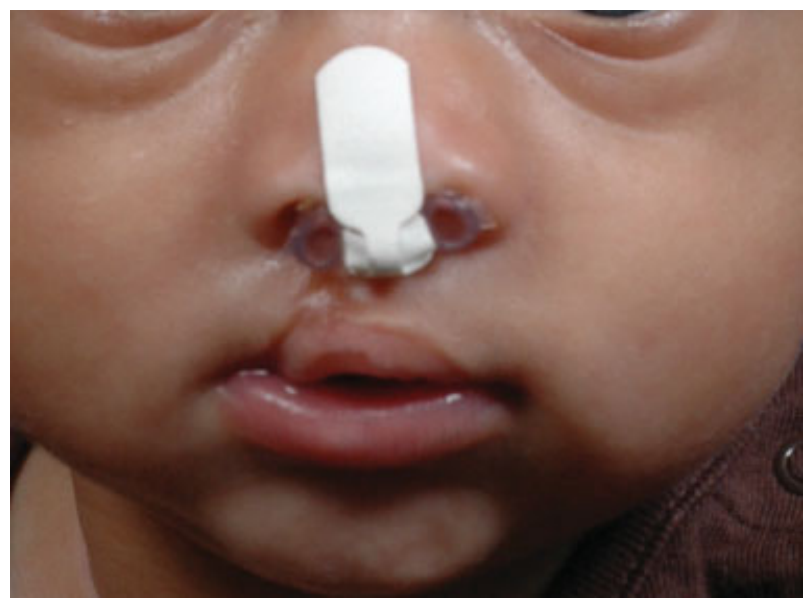

Fig. 7 Nasal stents are secured in the nostrils for 5 to 7 days with a transcolumellar suture, and then taped in place for up to 6 weeks postoperatively. 
A

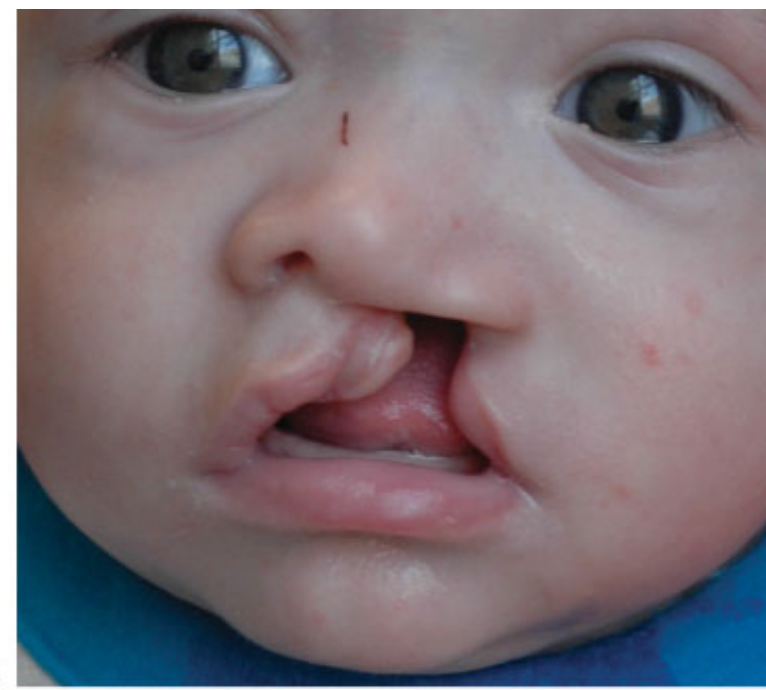

B

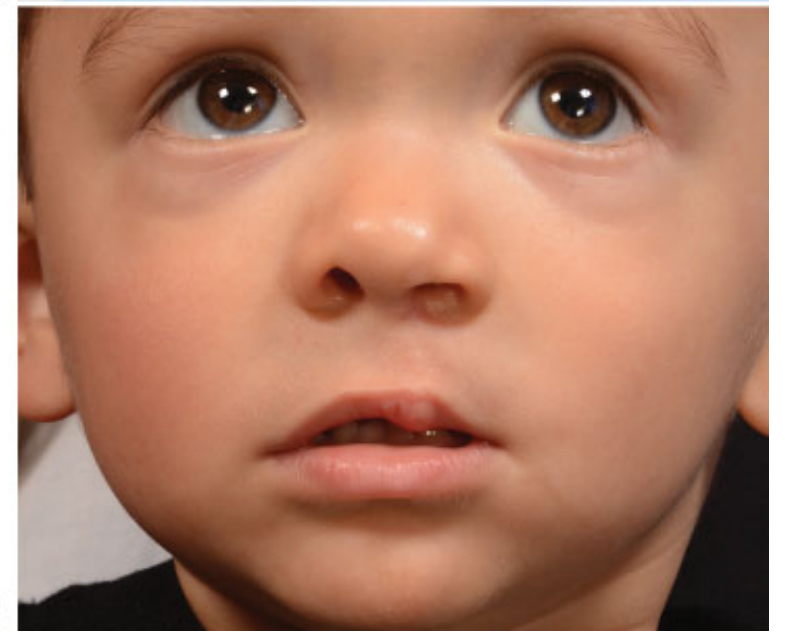

Fig. 8 Examples of anatomic subunit approaches for a complete cleft lip and palate. (A) Pre-op (same as - Fig. 4) and (B) 9 months postoperatively (note a small amount of excess vermillionmucosal junction and slight relapse of left alar hooding).

maxillary frenuloplasty is completed. Muscle closure begins with the inferior-most aspect of the cleft and noncleft orbicularis oris using a vertical mattress to evert the tubercle region. Four to five 4-0 Monocryl sutures are then orthogonally placed between the orbicularis oris margins with emphasis on choosing slightly more cephalic bites of the medial (cleft side) muscle, so as to lengthen the muscle layer height. These sutures are tagged and tied sequentially with the knots under the muscle to prevent subdermal suture abscesses. (Hint: Braided suture such as Vicryl seems to result in more stitch abscesses than PDS or Monocryl.)

The key skin closure is at the vermilion-cutaneous junction. The cutaneous (white) roll is first approximated using subcuticular 6-0 Monocryl sutures to "connect the dots" of the vermilion-cutaneous junction and cutaneous roll tattoos from the cleft and noncleft sides. The lateral lip triangular flap back cut is made perpendicular to the vertical philtral column, starting at the tattooed points 8 to 9 (just above the cutaneous roll). Deep dermal sutures complete the skin closure. The vermillion closure and the laterally based vermillion triangle flap are inset with 7-0 Vicryl sutures, while the mucosa is trimmed to attain symmetry and everted with 5-0 chromic sutures.

Dermabond is applied to the skin in three thin layers. Silicone nasal conformers are trimmed, placed into the nostrils, and secured with a transeptal suture (4-0 nylon) (-Fig. 7). They are removed after 7 days, after which the stents are used for up to 6 weeks with daily cleaning and taping onto the nasal tip. Immediate results can have slight shortening of the lip height from wound contraction, but resolves over the first year. Relapse of nostril hooding over the first year is possible, thus supporting slight overcorrection (-Fig. 8).

\section{Conclusion}

Fisher introduced the subunit technique for unilateral cleft lip repair to a widespread audience in $2005 .{ }^{1}$ The technique has been adopted by surgeons in North America and has greatly expanded. In a 2008 survey, 46\% of North American surgeons used a rotation-advancement technique or common hybrid, while triangle flap repairs were the second most common (9\%; which at that time were mostly Randall Tennison and then Fisher anatomic subunit repair). ${ }^{11}$ As I have progressed in my comfort level with the subunit techniques, I routinely called upon my rotation-advancement principles. The transition can be easier by starting with the measurements and landmarks that you already use and extrapolating the similarities to the subunit approach. Hopefully, some of the suggestions that I learned through experience will be of use to readers as they expand their cleft lip repair approaches.

\section{References}

1 Fisher DM. Unilateral cleft lip repair: an anatomical subunit approximation technique. Plast Reconstr Surg 2005;116(1):61-71

2 Tollefson TT, Sykes JM. Unilateral cleft lip. In: Goudy S, Tollefson TT, eds. Complete Cleft Care. New York, NY: Thieme; 2015:37-62

3 Tennison CW. The repair of the unilateral cleft lip by the stencil method. Plast Reconstr Surg (1946) 1952;9(2):115-120

4 Mohler LR. Unilateral cleft lip repair. Plast Reconstr Surg 1987; 80(4):511-517

5 Cutting CB, Dayan JH. Lip height and lip width after extended Mohler unilateral cleft lip repair. Plast Reconstr Surg 2003;111(1): 17-23, discussion 24-26

6 Noordhoff MA. The Surgical Technique for the Unilateral Cleft LipNasal Deformity. Taipei: Noordhoff Craniofacial Foundation; 1997

7 Tajima S, Maruyama M. Reverse-U incision for secondary repair of cleft lip nose. Plast Reconstr Surg 1977;60(2):256-261

8 Tollefson TT, Senders CW, Sykes JM. Changing perspectives in cleft lip and palate: from acrylic to allele. Arch Facial Plast Surg 2008; 10(6):395-400

9 Matsuo K, Hirose T. Preoperative non-surgical over-correction of cleft lip nasal deformity. Br J Plast Surg 1991;44(1):5-11

10 Skoog T. A design for the repair of unilateral cleft lips. Am J Surg 1958;95(2):223-226

11 Sitzman TJ, Girotto JA, Marcus JR. Current surgical practices in cleft care: unilateral cleft lip repair. Plast Reconstr Surg 2008;121(5): 261e-270e 